Technical Reports

\title{
Analysis of Magnetic Wheel Adhesion Force for Climbing Robot
}

\author{
${ }^{1}$ Haydn Welch and ${ }^{2}$ Shyamal Mondal \\ ${ }^{1}$ Division of Mechanical Engineering, London Southbank University, 103 Borough Road, London, SE1 OAA, UK \\ ${ }^{2}$ Division of Mechanical Engineering, London Southbank University, 103 Borough Road, London, SE1 OAA, UK
}

\section{Article history}

Received: 08-08-2019

Revised: 10-08-2019

Accepted: 30-08-2019

Corresponding Author:

Shyamal Mondal

Mechanical Engineering,

London South Bank University,

United Kingdom

Email: mondals@1sbu.ac.uk

\begin{abstract}
This paper describes the aim of designing, building and testing magnetic wheel based an autonomous climbing robot, for use in conjunction with Non-Destructive Testing (NDT) inspection on vertical towers. Through extensive review of previous generations of climbing robot, a hybrid wheel and permanent magnetic adhesion system has been designed and discussed in this paper. Using mathematical modelling and Finite Element Analysis (FEA) of differing magnet geometries, an adhesion system has been developed to produce the required amount of adhesion force and has been empirically tested at several intervals are presented in this paper. To complement this adhesion system, a lightweight, cost effective body is designed using 3D CAD software and manufactured using rapid prototyping methods. This has been done to incorporate the electronic equipment used to sense the working environment, drive the robot and carry equipment capable of performing defect detection tasks. To do this, a range of sensors, motors and auxiliary equipment has been used and controlled by a microcontroller. Finally, a functional scale prototype are manufactured, assembled and tested on a cylindrical test rig that closely imitated its intended work environment.
\end{abstract}

Keywords: Adhesion Force, Robotics, Magnetic Wheel

\section{Introduction}

Autonomous climbing robots are robotic systems capable of traversing multiple planes, inclined or otherwisea and have been implemented in every sector of engineering from inspection of military submarines to space crafts. As many robotics engineers have been known to take their inspiration from nature, there are many variants of climbing robots using astounding techniques to fulfil ever expanding niches. As well as these ingenious adhesion methods and body designs, as technology improves, these robotic systems are being fitted with a plethora of environment detecting sensors to further increase their desirability within industry and pave the way for the future of the engineering sector.

As with many engineering endeavors, the use of robotics within industrial applications are primarily to make their human counterparts job safer, more efficient and easier to do. The reason this technology is being pioneered is to remove human workers from potentially harmful or even life-threatening tasks and replace them with more expendable, programmable robotic systems. It is also the intention to eliminate systematic error from vital inspection related tasks, this being a considerable contributing factor to failure. It is the aim of this work to design and build an autonomous climbing robot fitted with appropriate sensors for material defect detection by means of Non-Destructive Testing (NDT). This robot is built with the intention of carrying out defect detection on wind turbine towers and is therefore be fully capable of both climbing vertical ferromagnetic surfaces and accurately detecting any possible flaws within the tower itself.

To ascertain the suitability of the adhesion system that will adhere the prototype to the work environment, before the manufacturing process, simulations has been compiled to analyses the forces produced by magnets of varying geometries. Through calculation, these magnet's adhesion forces with differing air gaps has then been assessed and the most suitable is being used for the manufacture of the final prototype. With the chosen adhesion system proposed, simulated and analyzed, the final, prototype has been tested to confirm that the theory accurately describes the chosen adhesion method and that the prototype functions as intended. 


\section{Technical Background and Context}

For the design of a climbing robot, research into adhesion methods is of the highest importance. In this paper, three adhesion methods shall be evaluated for use on the prototype proposed: permanent magnetic, bioinspired and electrostatic adhesion. (Guo et al., 2015).

Unver and Sitti, (2009) created a climbing robot that utilises synthetic dry adhesives. Tankbot uses a "flat bulk, tacky elastomer" known as Vytaflex V10 (Smooth-On) as its form of adhesion. It is a light, agile and efficient robot capable of traversing not only horizontal and vertical surfaces but inverted surfaces too. It can do this on many surfaces such as: glass, acrylic, brick, wood and metal.

Prahlad et al. (2008) present a robot that uses electrostatic adhesion and accomplishes this with the aid of electro-adhesive flaps incorpartaed into dual tracks. This technology lends advantages such as: adaptable clamping to a variety of surfaces, low power consumption, resistant to external contamination, and quick, controllable attachment/detachment.

San-Millan (2015; Wu et al., 2012) presents three ways with which permanent magnet adhesion can be implemented. The first two involve standard wheels integrated with several cylindrical magnets. The way they differ is in the magnet arrangement, one is perpendicular relative to the central axis, the other is orientated in a horizontal fashion relative to the central axis and is enclosed by a flux plate. There are two problems that exist for these methods, one is that only a small percentage of the magnets are sufficiently close to the surface. The other relates to the physical size of the wheel, if more force is required the size and mass must increase. These issues could be resolved by: an externally orientated set of magnets or an optimisation of the layout of the magnets. The previously mentioned resolutions lead to the third method of magnetic adhesion presented in this paper. The Halacha array is an arrangement of magnets that is orientated in such a way that the magnetic adhesion forces are maximised (Stepson et al., 2017).

Symmetrically centralised magnetic wheel unit for wall climbing robots have been proposed by Kang Liu (2007) and the others. The unit consists of wheel, permanent magnetic blocks and connecting parts. The unit has a fixed number of permanent magnets symmetrically arranged around the wheel and there is always a certain air-gap between the magnet blocks and the surface. The characteristics of the design provides stable adsorption for between the magnetic blocks and the surface with different curvature radius. The tracked type mechanism has large adsorption area and great adhesion force due to the small gap between the track magnets and the ferromagnetic surface when active. Even though, it can rotate about its axel; it is not really flexible as the wheeled type thus making it harder to turn (Dethe and Jaju, 2014; Jones and Flynn, 1998; Shen and Gu, 2005; Liu and Zhang, 2007).

In summary, the literature resource has been showed that while every method of adhesion has both pros and cons, some are more applicable to this design than others. For this reason, it has been decided that wheels integrated with permanent magnets will be used as a form of both adhesion and locomotion. It has also implied the importance of lightweight, yet strong materials, hence the material to be used in this application will be acrylic. Another advantage to using materials such as this is the ease with which it can be cut to specification, this material is easily cut by a laser cutter which happens to be a very cheap method of rapid prototyping. Finally, a peeling tail will be incorporated, this is because in one way or another, almost every paper reviewed made comment to the importance of its application.

\section{Simulations}

Using a piece of finite element analysis software; FEMM, the designed wheel within which the adhesion system will be integrated could have its potential magnetic adhesion forces simulated. This process involved defining things such as: materials, boundary conditions and magnetic force vectors and once this had been done the simulation could commence.

For use in this design, magnetic field strength was the property required to calculate produced magnetic forces. For this reason, a comparison between $6 \mathrm{~mm}$ and $8 \mathrm{~mm}$ diameter magnets; both with a thickness of $3 \mathrm{~mm}$, were simulated. In addition to this, simulations were run with the magnets both parallel and at an angle to the plate. These two simulations would provide a maximum and minimum produced force. This assumption could be made as maximum force occurs when the magnet is parallel to and touching faces with the steel plate as shown in Fig. 1 and 3. Equally, minimum magnetic force occurs at the distance where a parallel face is at its greatest distance from the plate, in this case, this occurred at $12^{\circ}$ as shown in Fig. 2 and 4. The results of the simulations have done with 6 and $8 \mathrm{~mm} \mathrm{~N} 35$ neodymium eclipse magnets.

With the simulations complete and the theorized adhesion forces analyzed, the calculations; as seen below, can be used to calculate an appropriate air to be incorporated into the adhesion systems design. An air gap was required to provide the required amount of adhesion forces whilst refraining from causing damage to the test surface via physical contact. 

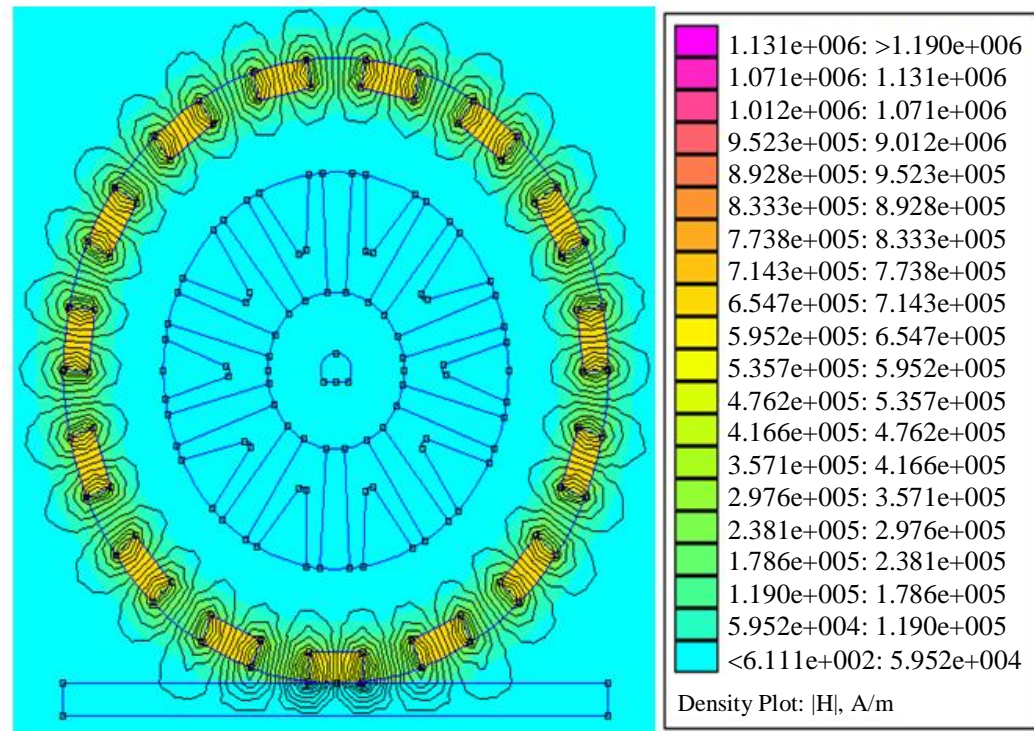

Fig. 1: 2d Simulated magnetic field strength with $6 \mathrm{~mm}$ magnet parallel with the steel plate
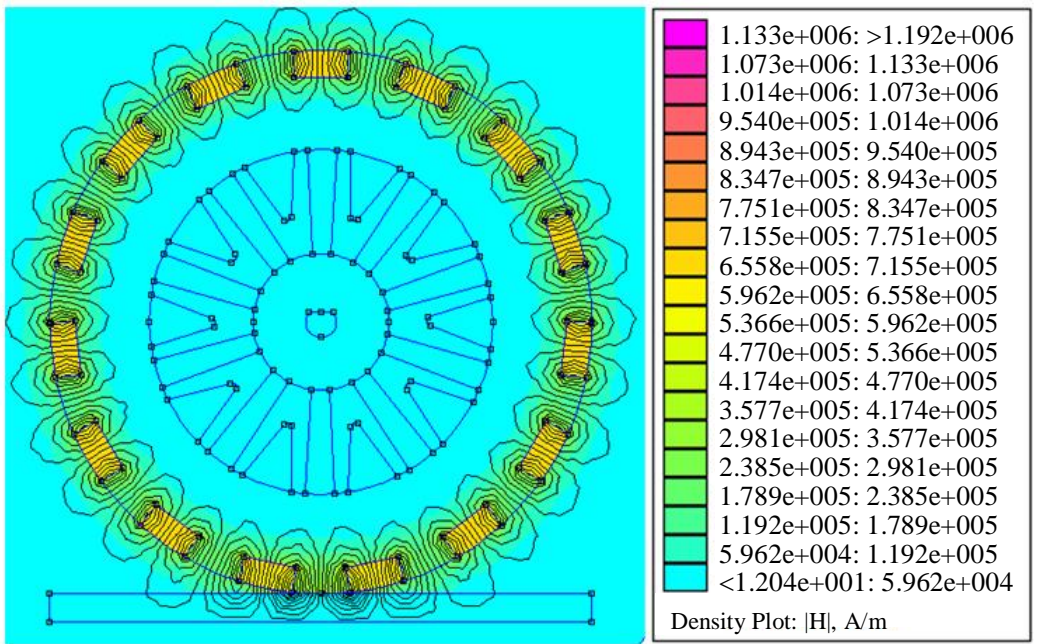

Fig. 2: $2 \mathrm{~d}$ Simulated magnetic field strength with $6 \mathrm{~mm}$ magnet at $12^{0}$ from the steel plate

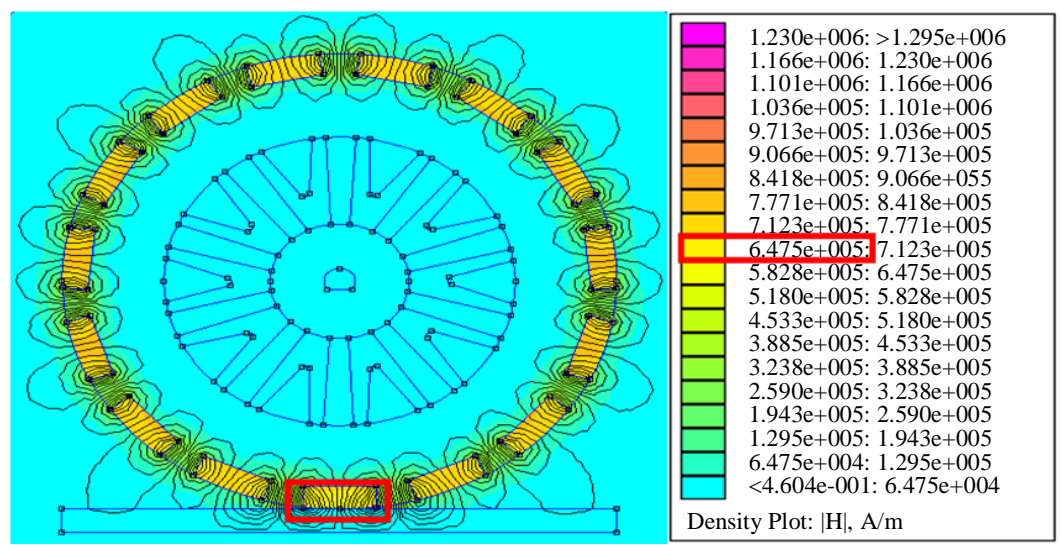

Fig. 3: 2d Simulated magnetic field strength with $8 \mathrm{~mm}$ magnet parallel with the steel plate 


\section{Calculations and Graphs}

In the case of Fig. 4, a value of $6.325 \times 10^{5} \mathrm{~A} / \mathrm{m}$ was used. Using the equation below (Tavakoli et al., 2013), values for magnetic force could be calculated for both the 6 and $8 \mathrm{~mm}$ magnets at both orientations:

$$
F_{\text {Magnetic }}=\frac{H^{2} \times A \times \mu}{2}
$$

Where:

$F_{\text {Magnet }}=$ Force produced by magnets at a distance of 0 $\mathrm{mm}(\mathrm{N})$

$H \quad=$ Magnetic field strength

$A=$ Area of magnet in contact with the steel plate:

$$
\begin{aligned}
& \mu=\text { Permeabillity of air }=4 \pi \times 10^{-7} \\
& F_{8 \text { mm max }}=13.241 \mathrm{~N} \\
& F_{8 \text { mm min }}=12.635 \mathrm{~N} \\
& F_{6 \text { mm max }}=10.637 \mathrm{~N} \\
& F_{6 \text { mm min }}=7.710 \mathrm{~N}
\end{aligned}
$$

At orientations providing maximum and minimum forces:

Using these values for magnetic forces and the equation below (Clarke, 2018), forces with varying air gaps were calculated:

$$
F_{\text {Air gap }}=\frac{F_{\text {Magnetic }}}{1+S^{3}}
$$

Where:

$F_{\text {Air gap }}=$ Magnetic force altering air gap (N)

$S \quad=$ Air gap between magnet and steel plate $(\mathrm{mm})$

Using Matlab, firstly the pull forces provided by the supplier were plotted to give some vales to compare the obtained results to, this can be seen in Fig. 5.

With the figures for magnetic forces with varying air gaps, as provided by supplier, as a datum, the results acquired by the simulations and calculations could be compared to highlight their accuracy. The results obtained by simulation and calculation can be seen in Fig. 6.

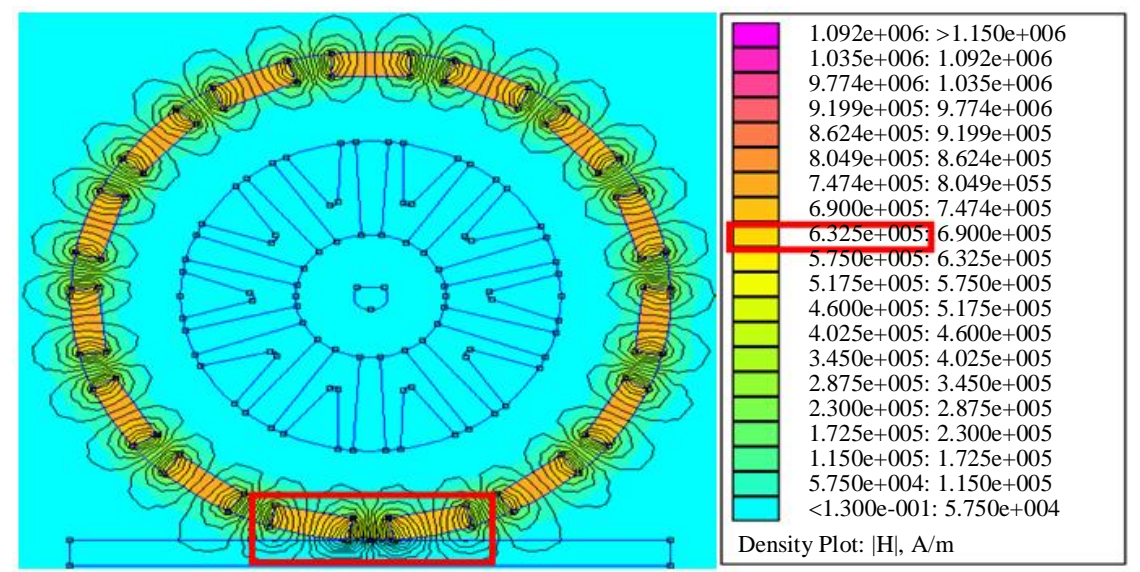

Fig. 4: 2 d Simulated Magnetic Field Strength with $8 \mathrm{~mm}$ magnet at $12^{0}$ from the steel plate

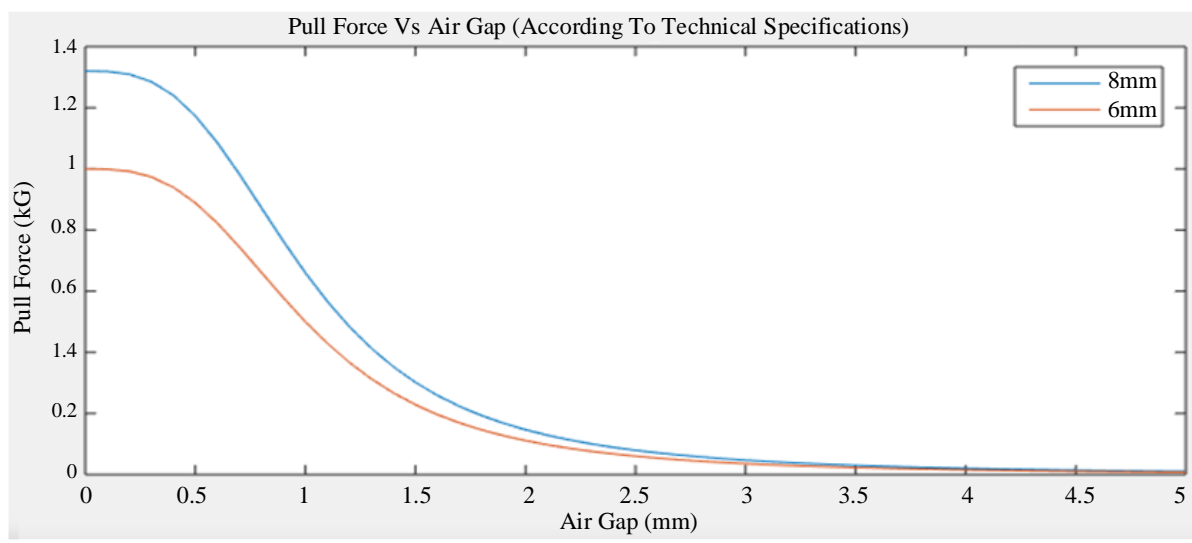

Fig. 5: Pull force with varying air gaps as provided by supplier 


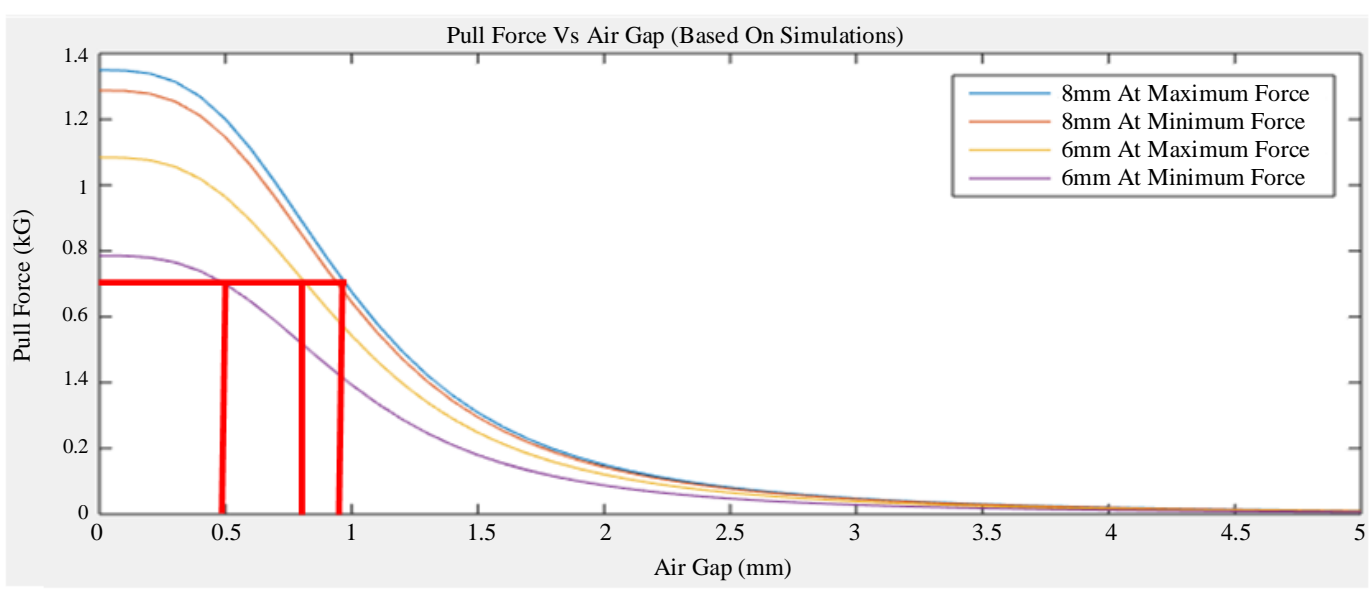

Fig. 6: Pull force with varying air gaps as simulated

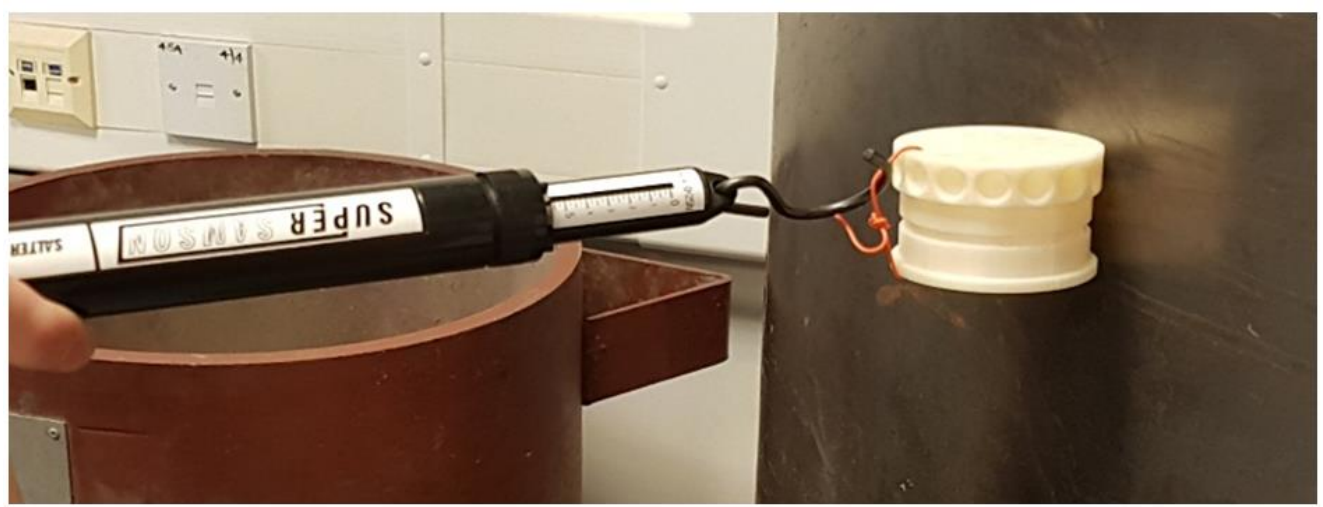

Fig. 7: Testing adhesion forces with no air gap. Using Newton meter

Using the forces provided by the minimum and maximum values according to the simulations, the forces with varying air gaps were calculated and plotted, as seen in Fig. 6. Using $0.7 \mathrm{~kg}$ as an estimate of the overall weight of the robot, this value was used to cross reference the required air gap between the magnets and the steel plate. Using the values provided by the simulations and having analyzed Fig. 6, a decision was made to use the $8 \mathrm{~mm}$ neodymium magnets. This decision was made because it was felt that using the $8 \mathrm{~mm}$ magnets would provide a little more flexibility in the design with respect to air gaps. For example, due to the nature of the wheel; it being circular, to have to the magnets flush with the lowest point of the slot in which they sit, an air gap of $0.3 \mathrm{~mm}$ is already present. This $0.3 \mathrm{~mm}$ air gap is already nearing the minimum air gap that would be required should $6 \mathrm{~mm}$ magnets be used. In addition to this, the results obtained from the simulation of the $8 \mathrm{~mm}$ magnets seem to show less variation in the maximum and minimum values, leading to the belief that these magnets would be more suitable for this purpose.

\section{Test of Adhesion Forces}

To verify the generated adhesion forces and the results obtained by means of simulations, a simple test was carried out as shown in Fig 7. Using a Newton meter, the wheel; integrated with an array of 15 magnets, was attached to the steel plate test rig and the force required to remove the wheel from the wall was recorded as shown in Fig 8. The first, of this two-part test, consisted of testing the adhesion forces generated with no air gap present. To do this the magnets were incorporated into the wheel with no elastomer track present on the wheel. The wheel was then attached to a Newton meter and the wheel was orientated in such a way that one magnet was parallel to the steel plate. The wheel and Newton meter were then pulled until the wheel became detached from the plate, the value of force at which this occurred was then recorded. To simulate both the maximum and minimum forces the wheel was then orientated leaving a $12^{\circ}$ angle between two magnets and the steel plate. Again, the wheel was pulled until detachment and the force was recorded. 


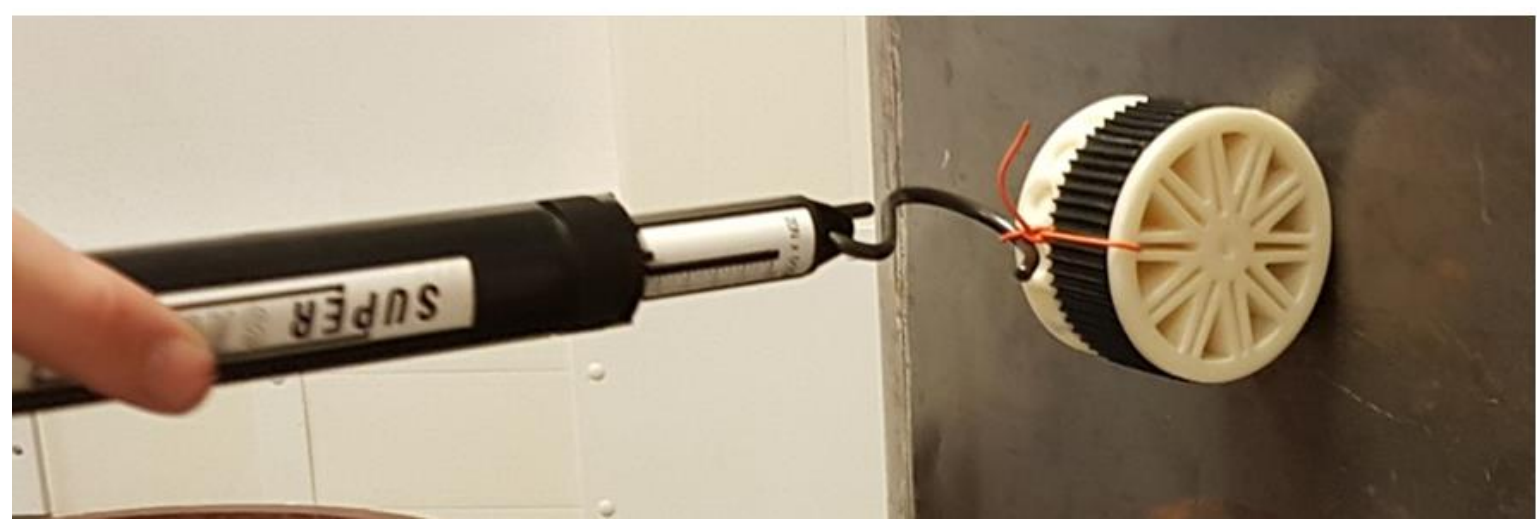

Fig. 8: Testing adhesion forces with a $0.7 \mathrm{~mm}$ air gap. Using Newton meter

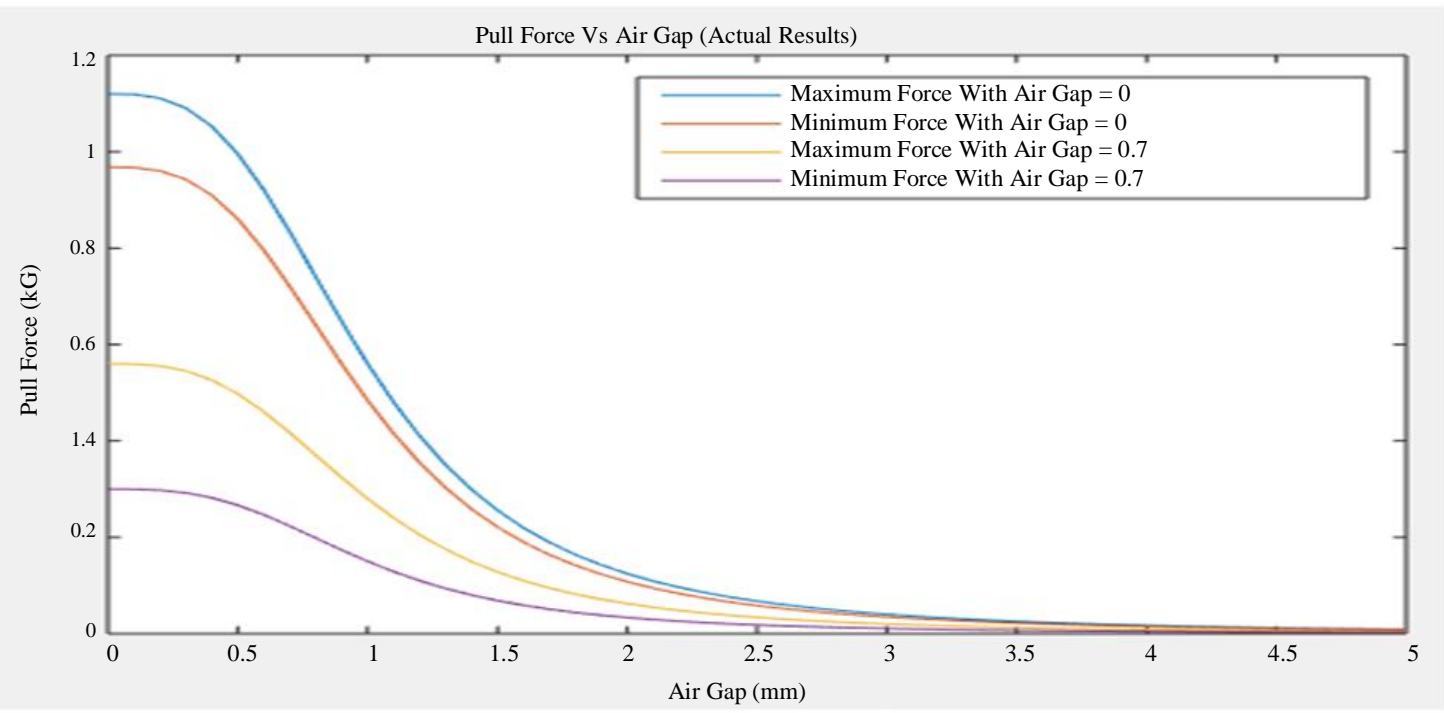

Fig. 9: Results from test with Newton meter

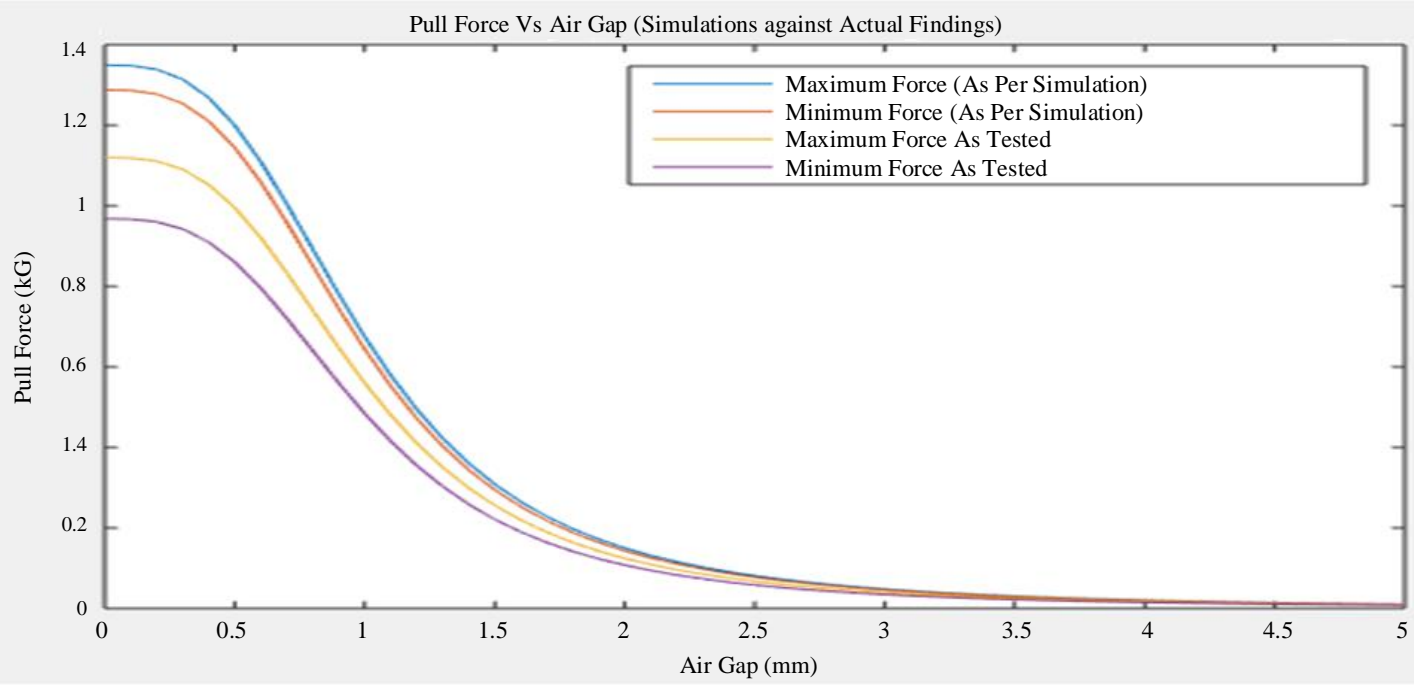

Fig. 10: Results from test with Newton meter vs simulated results from same magnets 


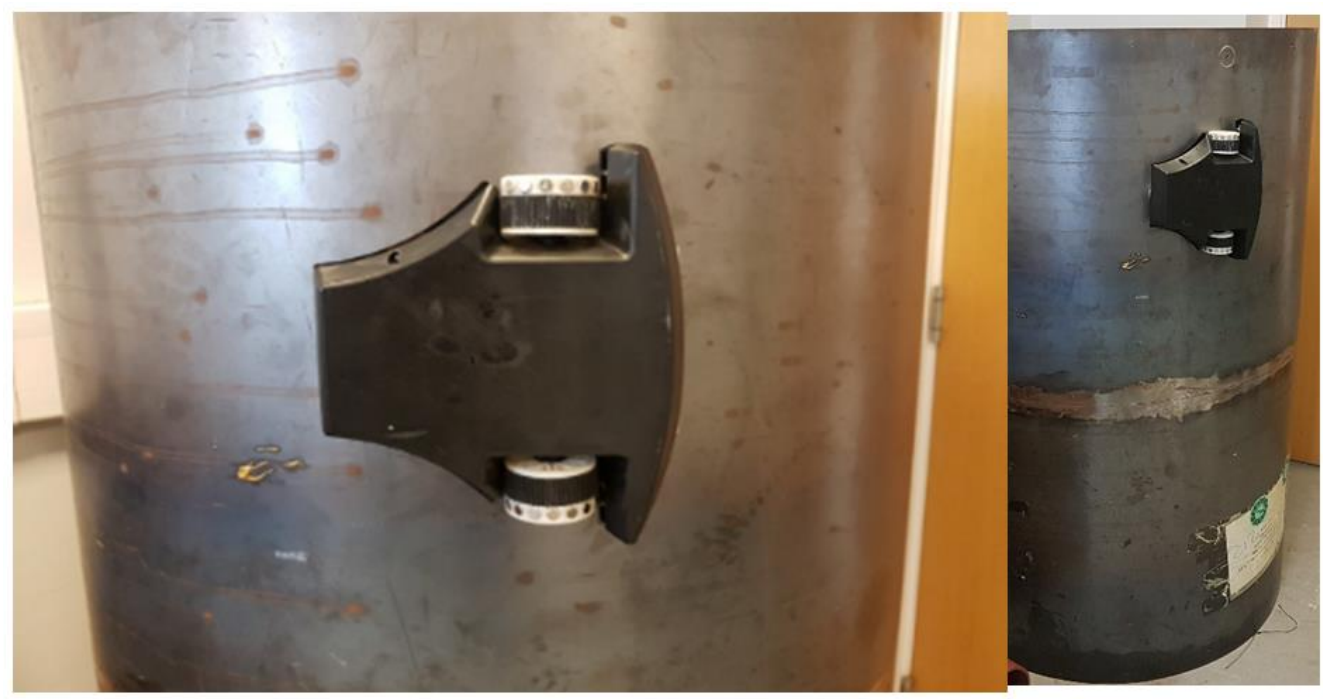

Fig. 11: Test of prototype on cylindrical test rig

To now simulate the magnetic forces generated with an air gap present, the elastomer track was added and this provided an air gap of $0.7 \mathrm{~mm}$. As before, the two tests were repeated and again both sets of results were recorded and plotted, see Fig. 9.

It can be seen in Fig. 10 that the results obtained from the test were of a very comparable nature to those of the values acquired by means of simulation. This suggests that both the simulation was of an accurate nature and that both the conclusions drawn from the results of the simulation and the magnets selected were indeed correct.

To test that the adhesion system was designed correctly, the fully assembled prototype was placed horizontally on the vertical cylindrical test rig, as seen below in Fig. 11. With the prototype remaining successfully adhered to the test surface, the final test was preformed to assess the success of the adhesion system with respect to the movement of the prototype around its working environment.

At this point the prototype is tested and the successful movement of the robot as it traversed around the test rig proved that the adhesion system designed for this application has in fact been successful in its aim to provide adequate adhesive forces to aid in a defect detection of wind turbine towers with the use of an autonomous robotic system.

\section{Results}

The results obtained over the duration of this test has been showed great promise and succeeded in providing yet more research into the ever-growing field of autonomous robotic systems utilizing permanent magnetic adhesion. It has been succeeded in producing a unique design that not only suitably housed the essential equipment required for NDT inspection, but did so in the most efficient way possible. In addition to this, through extensive FEMM simulation as well as mathematical modelling, the magnetic adhesion forces were calculated, simulated and were of a comparable nature to those obtained by both existing literature and by the supplier of the magnets in question.

Upon completion of the initial testing of the adhesion forces produced by each wheel integrated with an array of 15 magnets, empirical evidence confirmed the suitability of the chosen magnets. This was done as values of magnetic strength obtained from both suppliers and simulation were replicated by means of repeatable experimentation.

In addition to the successful testing of the adhesion forces and the proof of theory that the produced adhesion system indeed produces the estimated amount of force, the adhesion method was also proved adequate to adhere the prototype to the test surface. As well as simply adhering to the cylindrical test rig, the prototype also succeeded in maintaining adhesion during locomotion.

\section{Conclusion}

In summary, a suitable adhesion method capable of adhering an autonomous robot to vertical surfaces has been proposed. Through calculation and simulations, this adhesion method has been designed and developed to fulfil its intended purpose as efficiently and at as little cost as possible. In addition to this, the adhesion system has been tested at several intervals to verify the proposed system continues to function as designed and match the theory with repeatable empirical evidence. 
To conclude, a functioning prototype has been manufactured and tested for the intended purpose of carrying NDT inspection equipment on inclined ferromagnetic surfaces. In addition to this, the work presented in this paper provides a great amount of insight into the field of autonomous climbing robots utilizing permanent magnetic adhesion for NDT inspection.

\section{Acknowledgement}

The Authors are thankful to the robotics reserach center for providing the testing rigs and grateful to the technical team of London South Bank University for providing support.

\section{Author's Contributions}

Haydn Welch: He contributed to design the research plan, carry out all experiments and drafted the manuscripts.

Shyamal Mondal: He supervised this work and devised the main conceptual ideas. He provided feedback and helped to outline the exploration and manuscript.

\section{Ethics}

This paper has been neither published nor is under review elsewhere. There are no ethical issues involved.

\section{References}

Clarke, R., 2018. Magnetism: quantities, units and relationships.

Dethe, R.D. and S.B. Jaju, 2014. Developments in Wall Climbing Robots: A Review. Int. J. Eng. Res. Gener. Sci.

Guo, J., L. Justham, M. Jackson and R. Parkin, 2015. A concept selection method for designing climbing robots. Key Eng. Mater., 649: 22-29.

DOI: $10.4028 /$ www.scientific.net/KEM.649.22

Jones, J.L., and A.M. Flynn, 1998. Mobile Robots: Inspiration to Implementation. 2nd Edn., CRC Press ISBN-10: 1568810970, pp: 486.

Liu, K. and W. Zhang, 2007. Symmetrically centralized magnetic-wheel unit for wall-climbing robots. Proceedings of the 13th IASTED International Conference Robotics and Applications, (ICRA, 2007).
Prahlad, H., R. Pelrine, S. Stanford, J. Marlow and R. Kornbluh, 2008. Electroadhesive robots-wall climbing robots enabled by a novel, robust, and electrically controllable adhesion technology. Proceedings of the IEEE International Conference on Robotics and Automation, May 19-23, IEEE Xplore Press, Pasadena, CA, USA, pp: 3028-3033. DOI: 10.1109/ROBOT.2008.4543670

San-Millan, A., 2015. Design of a teleoperated wall climbing robot for oil tank inspeion. Proceedings of the 23rd Mediterranean Conference on Control and Automation (MED), June 16-19, IEEE Xplore Press, Torremolinos, Spain, pp: 255-261. DOI: 10.1109/MED.2015.7158759

Shen, W. and J. Gu, 2005. Permanent magnetic system design for the wall-climbing robot. Proceedings of the IEEE International Conference on Mechatronics Automation Niagara Falls, July 29, IEEE Xplore Press, Niagara Falls, Ont.

DOI: 10.1109/ICMA.2005.1626883

Stepson, W.A.V., A.D.I.M. Amarasinghe, P.N.R. Fernando and Y.W.R. Amarasinghe, 2017. Design and development of a mobile crawling robot with novel halbach array based magnetic wheels. Proceedings of the IEEE/RSJ International Conference on Intelligent Robots and Systems (IROS), Sept 24-28, IEEE Xplore Press, Vancouver, BC, Canada, pp: 6561-6566.

DOI: $10.1109 /$ IROS.2017.8206567

Tavakoli, M., C. Viegas, L. Marques, J.N. Pires and A.T. de Almeida, 2013, Magnetic omnidirectional wheels for climbing robots. Proceedings of the IEEE/RSJ International Conference on Intelligent Robots and Systems, Nov. 3-7, IEEE Xplore Press, Tokyo, Japan, pp: 266-271. DOI: 10.1109/IROS.2013.6696363

Unver, O. and M. Sitti, 2009. Tankbot: A miniature, peeling based climber on rough and smooth surfaces. Proceedings of the IEEE International Conference on Robotics and Automation, May 1217, IEEE Xplore Press, Kobe, Japan, pp: 2282-2287. DOI: 10.1109/ROBOT.2009.5152304

Wu, M., G. Pan, T. Zhang, S. Chen and F. Zhuang et al., 2012. Design and optimal research of a non-contact adjustable magnetic adhesion mechanism for a wallclimbing welding robot. Int. J. Adv. Robo. Syst. DOI: $10.5772 / 54008$ 